\title{
Expert consensus on clinical applications of high-frequency oscillations in epilepsy
}

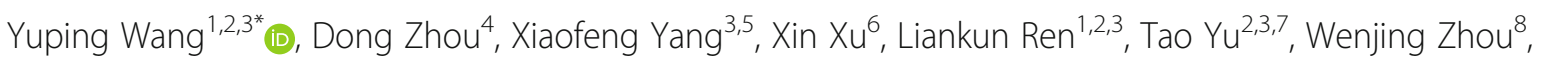
Xiaoqiu Shao 9,10 , Zhixian Yang ${ }^{11}$, Shuang Wang ${ }^{12}$, Dezhi Cao ${ }^{13}$, Chunyan Liu ${ }^{1,2,3}$, Shang Yeong Kwan ${ }^{14}$ and Jing Xiang ${ }^{15,16}$

\begin{abstract}
Studies in animal models of epilepsy and pre-surgical patients have unanimously found a strong correlation between high-frequency oscillations ( $\mathrm{HFOs},>80 \mathrm{~Hz}$ ) and the epileptogenic zone, suggesting that HFOs can be a potential biomarker of epileptogenicity and epileptogenesis. This consensus includes the definition and standard detection techniques of HFOs, the localizing value of pathological HFOs for epileptic foci, and different ways to distinguish physiological from epileptic HFOs. The latest clinical applications of HFOs in epilepsy and the related findings are also discussed. HFOs will advance our understanding of the pathophysiology of epilepsy.
\end{abstract}

Keywords: High-frequency oscillations, Ripple, Fast ripple, Interictal spike, Epilepsy, Biomarker

\section{Background}

High-frequency oscillations (HFOs) have become a new biomarker of epileptogenic zone in the brain. This expert consensus highlights the development of clinical applications of HFOs in the context of epilepsy as well as the findings, in the aim to provide guidance for epilepsyrelated clinical use of HFOs.

\section{Definition of HFOs and their standard detection techniques}

Definition of HFOs

HFOs are oscillatory high-frequency $(>80 \mathrm{~Hz})$ brain signals recorded by electroencephalography (EEG) due to the transient local-field potential (LFP) oscillations. HFOs are defined as having at least four oscillations with sinusoidal-like morphology in the filtered signal $(>80 \mathrm{~Hz})$ with a root mean square amplitude increase of more than 5 times the standard deviation compared to background brain activity $[1,2]$ or

\footnotetext{
* Correspondence: wangyuping01@sina.cn; doctorwangyuping@163.com 'Department of Neurology, Xuanwu Hospital, Capital Medical University, Beijing 100000, China

${ }^{2}$ Beijing Key Laboratory of Neuromodulation, Beijing 100000, China Full list of author information is available at the end of the article
}

with energy larger than the 95 percentile of the surrounding background [3].

\section{Classification of HFOs by frequency}

The definition of HFO frequency varies among studies. Narrowly, HFOs are considered to have a frequency range of $80 \mathrm{~Hz}$ to $500 \mathrm{~Hz}$, and are classified into two common subtypes according to the frequency: ripples $(80-250 \mathrm{~Hz})$ and fast ripples $(250-500 \mathrm{~Hz})$. More broadly, HFOs are defined as brain activities with frequencies higher than $30 \mathrm{~Hz}$, and divided into four sub-bands: low gamma band $(30-80 \mathrm{~Hz})$, high gamma band $(80-150 \mathrm{~Hz})$, ripples $(80-250 \mathrm{~Hz})$ and fast ripples $(250-500 \mathrm{~Hz})$ band [4]. Of note, the high gamma band can overlap with the ripple band.

Recently, very-high-frequency oscillations (VHFOs, $>600$ $\mathrm{Hz}$ ) have been reported in patients with epilepsy $[5,6]$. VHFOs can be classified into very-fast ripples (500-1 000 $\mathrm{Hz}$ ) and ultrafast ripples $(1000-2000 \mathrm{~Hz})$.

\section{Possible mechanisms of HFOs}

The synchronizing mechanisms of HFOs need to be fast enough to synchronize activity in 2 to $5 \mathrm{~ms}$. Possible mechanisms of HFOs include ephaptic interactions [7], electrotonic

(c) The Author(s). 2020 Open Access This article is licensed under a Creative Commons Attribution 4.0 International License, which permits use, sharing, adaptation, distribution and reproduction in any medium or format, as long as you give appropriate credit to the original author(s) and the source, provide a link to the Creative Commons licence, and indicate if changes were made. The images or other third party material in this article are included in the article's Creative Commons licence, unless indicated otherwise in a credit line to the material. If material is not included in the article's Creative Commons licence and your intended use is not permitted by statutory regulation or exceeds the permitted use, you will need to obtain permission directly from the copyright holder. To view a copy of this licence, visit http://creativecommons.org/licenses/by/4.0/. 
coupling via gap junctions [8], and fast synaptic transmission [9]. It is now considered that HFOs reflect summation of mainly principal cell action potentials from fast-firing neurons which are hypersynchronized through fast synaptic transmission or gap junction coupling $[10,11]$. There is evidence that interictal and preictal discharges are mediated by different cellular and synaptic mechanisms [12].

Although ripples in field potentials are linked with neuronal depolarization and firing, especially from fast-rhythmicbursting neurons, $\mathrm{GABA}_{\mathrm{A}}$ inhibition also participates in the precise timing of firing during ripple oscillations [13]. However, individual pyramidal cells cannot fire fast enough to produce synchronized oscillations up to fast ripples. Rather, the fast ripples are produced from the intrinsic fastsynchronizing behavior of local damaged neurons, leading to the out-of-phase firing of neuronal clusters [14].

\section{Criteria of recording and analysis of $\mathrm{HFO}$ HFOs in intracranial EEG recording}

Different types of intracranial electrodes could record HFOs, including microelectrodes and macroelectrodes (depth or subdural).

HFOs are transient LFP oscillations [15, 16]. They can be similarly detected with microelectrodes (surface area: $150 \mu \mathrm{m}^{2}$ ) [17] and clinical macroelectrodes (surface area: $1-10 \mathrm{~mm}^{2}$ ) [18]. Microelectrodes record LFP from a volume of $\sim 1 \mathrm{~mm}^{3}$, while clinical macroelectrodes presumably record the activity from $\sim 100 \mathrm{~mm}^{3}$ of brain tissue. Oscillations detected with clinical macroelectrodes span a wide range of frequencies $(\sim \mathrm{DC}$ [direct current] -600 $\mathrm{Hz}$ ) [18]. The features of HFOs are related to the type of recording electrode.

The EEG must be sampled above 4 times of the interest upper frequency, in order to form the wave shape [19]. To record HFOs that include fast ripples with a frequency as high as $500 \mathrm{~Hz}$, a sample frequency of $2000 \mathrm{~Hz}$ or above should be attained. The amplifier with $0.1-500 \mathrm{~Hz}$ wide bandwidth and above 128 channels is recommended. A referential montage of epidural reference far from the suspected epileptogenic zone is used during recording [20]. To record HFOs, bipolar montages are recommended as they can neutralize the artifacts carried by two adjacent electrodes [21].

To identify HFOs, channels are displayed with the maximum time resolution on a computer monitor ( $\sim 0.6 \mathrm{~s} /$ page) to show all samples (1 200 samples of a signal sampled at $2000 \mathrm{~Hz}$ ). The amplitude scale is changed to $1 \mu \mathrm{V} / \mathrm{mm}$. The EEG signal is filtered at $80-250 \mathrm{~Hz}$ to display ripple events and at $250-500 \mathrm{~Hz}$ to display fast ripple events. Only events including at least 4 continuous oscillations are regarded as HFOs. Those separated by at least two non-HFO oscillations are considered to be two different events [22].

\section{HFOs in surface EEG recording}

Previously, the detection of HFOs was limited to intracranial EEG recording, but in recent years, it has extended to scalp EEG. Most studies reported that the scalp HFOs can be recorded mainly in ripple and gamma bands $(40-80 \mathrm{~Hz})$, but it has been suggested that the fast ripples are also detectable in scalp EEG [23]. It is difficult to define the relationships between scalp and intracranial HFOs, simultaneous scalp and intracranial recordings suggested that scalp HFOs may reflect cortical HFOs [24]. HFOs on scalp recording tend to have lower frequencies, and only high-amplitude cortical HFOs are detectable over the scalp [25]. The difficulty of scalp recording is the separation of low-amplitude HFOs from electromyography (EMG) artifacts [26]. The scalp HFOs should be confirmed by time-frequency analysis on filtered EEG traces [27]. In time-frequency analysis, muscle activity always has irregular shapes, so the HFOs can be morphologically distinguished from EMG artifacts [25].

In pediatric epilepsy, scalp EEG ripple oscillations have been detected in patients with continuous spikewaves during slow-wave sleep (CSWS), Landau-Kleffner syndrome (LKS) and related diseases, and ripple oscillations have discrete spectral components in the in time-frequency spectra [28]. HFOs are found to be more prevalent in atypical benign partial epilepsy (ABPE) than in benign childhood epilepsy with centrotemporal spikes, and more sensitive to methylprednisolone treatment than the spikes in ABPE [29]. Subsequently, by analyzing the HFOs in CSWS patients with different etiologies, researchers found that after methylprednisolone treatment the percentage reduction of HFOs is higher in patients with a genetic/ unknown etiology than in those with a structural etiology, the distribution of HFOs is focal while that of spikes is diffuse, and HFO-dominant regions are concordant with magnetic resonance imaging (MRI) abnormalities in structural CSWS patients [30]. HFOs seem to be a good biomarker to predict seizure and cognitive outcomes after methylprednisolone treatment in CSWS patients [31]. The amplitude of scalp ripples is at most about $10 \mu \mathrm{V}$.

\section{HFOs in Magnetoencephalography (MEG) recording}

MEG has been used in the detection of HFOs for nearly two decades [32-34]. HFOs, rather than spikes, are closely related with seizure severity and surgical outcomes [35, 36]. The interictal HFOs in MEG recordings are consistent with those in intracranial recordings in patients receiving epilepsy surgery [26]. Therefore, as a noninvasive approach to detecting HFOs, MEG can be used in patients who are not surgical candidates [35-37]. 
A set of methods have been developed to detect HFOs with MEG [32, 33, 38, 39]. To automatically detect HFOs, artificial intelligence (AI) such as stacked sparse autoencoder (SSAE) has been used to detect and classify HFOs in MEG data [40]. An SSAE-based MEG HFOs (SMO) detector has been developed to facilitate the clinical detection of HFOs. In fact, HFOs can also be localized and quantified with accumulated source imaging [39]. Virtual sensors (or virtual electrodes) are a technique that visualizes HFOs at the source level. Virtual sensors can eliminate the effect of head movement (head position is automatically corrected and registered to fiducial points). Spatiotemporal or "event-related" beamforming algorithms are adopted to localize origination of HFOs in interictal spikes by MEG recordings [41].

\section{Automatic detection of HFOs}

Since visual marking of HFOs is time-consuming and subjective, automatic detection is an essential alternative [26, 42-44]. Algorithms for detection of spontaneous HFOs commonly require at least four oscillations that stand out from the ongoing background activity, and at least $25 \mathrm{~ms}$ inter-event interval $[3,45]$.

Four automated methods of HFOs detection are widely used in recent years.

Automated method 1 The first method is based on the energy defined as the moving average of the root mean square amplitude of a filtered signal. Each channel is filtered and segments with energy above five times the standard deviation (SD) of the mean energy of the whole EEG and of more than $6 \mathrm{~ms}$ were considered as HFOs. This method has been reported to have a sensitivity of $84 \%$ [1].

Automated method 2 The second method is based on the short-time line length. Each channel is passed through a spectral equalizer (first-order differential filter), then band-pass filtered and the energy threshold is computed as the 95 percentile of the empirical cumulative distribution function for the epoch. Epochs lasted $3 \mathrm{~min}$ and included the possible events. This method has a sensitivity of $89.5 \%$ in a gamma band study. The specificity has not been reported, but in a previous study $\sim 85 \%$ of the candidate events were rejected as false positives [19].

Automated method 3 The third method is based on the Hilbert Transform, which is used to compute the signal envelope. Each channel is band-pass filtered and events are detected with a threshold five times the SD of the envelope over the whole EEG. The sensitivity is $100 \%$ with a specificity of $90.5 \%$ [46].
Automated method 4 The fourth method detects HFOs by using AI, which encodes the features of signals (e.g. morphology, amplitude, spatial, temporal, spectral, frequency and other signatures) [40]. The SMO detector has been developed to facilitate the clinical detection of HFOs. After configuration optimization, the newlydeveloped SMO detector outperforms other classic peer models by achieving $89.9 \%$ in accuracy, $88.2 \%$ in sensitivity, and $91.6 \%$ in specificity [40].

\section{Effects of physical condition on HFOs and the difference from artifacts}

HFOs occur more frequently during sleep, particularly in slow-wave sleep stage, than in the awake state [1].

The application of high-pass filters could results in the exposure of high-frequency components of sharp events and the occurrence of "false" ripple activity. It may be difficult to distinguish this "false" ripple activity from stand-alone oscillations sometimes [27]. Inadequate filter parameters also could result in artifacts and occurrence of false HFO. Using Fourier transform or wavelet transform to establish frequency power spectrum is helpful to solve this problem [47].

The channel with continuous artifacts could be discerned as the artifactual baseline activity was greater than that of other channels. The main frequency $(50 \mathrm{~Hz}$ or $60 \mathrm{~Hz}$ and harmonics) could also lead continuous artifacts, which can be easily identified by evaluating frequencies. Muscle artifacts always appear to be waxing and waning, and often occur on channels that close to skull. Reviewing suspected channels with epidural or extracranial channels simultaneously at $10-20 \mathrm{~s}$ per page will be helpful to reveal muscle artifacts, because this activity always occur at similar times [48]. In addition, if multiple channels have simultaneous HFOs, this electrical activity is likely to be artifact, one has to be cautious to mark it as epileptic. The main obstacle of HFO research is that various muscle activities usually result in a significant increase of gamma power $(>25 \mathrm{~Hz})$ and influence the signals recorded in HFO spectrum [3]. Application of bipolar montage may effectively reduce the contamination by EMG signals [49].

\section{The value of pathological HFOs in localizing epileptic foci}

Pathological HFOs are a new biomarker of the seizure onset zone

Pathological HFOs are considerd to be generated in the seizure onset zone (SOZ), or in the first-propagation zone. Analysis of HFOs may accelerate the identification and narrow down the location of the seizure-onset area compared to the routine EEG analysis of interictal and ictal epileptic activities. So pathological HFOs may be a new biomarker of SOZ [50]. 
HFOs can occur superimposed on spikes or in isolation. HFOs superimposed on spikes always have longer duration than those outside the spikes [51]. In general, the spatial extent of HFO zone were smaller than irritative zone, especially that of fast ripples. It has been suggested that HFOs can help discriminate between "green" and "red" spikes, which are markers of cortical areas of low and strongest epileptogenicity, respectively [52]. In contrast, the interictal spikes are more labile. Except occurring in the region of onset of spontaneous seizures, HFOs also occur in regions with a low threshold to generate after-discharges or evoked seizures in response to cortical stimulation, even outside the SOZ. This shows the correlation between HFOs with endogenous epileptogenicity [20].

HFOs are more specific in indicating the $\mathrm{SOZ}$ than spikes [51]. The ripples and fast ripples have a significantly higher occurrence rate and last significantly longer in the SOZ than outside [50]. Liu et al. demonstrated that HFOs in the SOZ have the highest degree of waveform similarity [53]. Determining with HFOs detection rate may have better sensitivity and specificity in identifying SOZ than with spikes detection rate [51]. The temporal lobe structure produces much more HFOs than the neocortex [20]. Different thresholds of HFOs rate can also be established to describe the SOZ of different types of epilepsy [51]. In addition, no difference has been found between the rate of HFOs in control neocortex and that in the non-seizure-onset zone of neocortex in patients with epilepsy [42].

Recently, there is a rising interest in studies combining HFOs with low-frequency oscillations $(<10 \mathrm{~Hz})$, even direct current (DC) shifts. Some studies reported that the co-occurrence of HFOs with $3-4 \mathrm{~Hz}$ delta waves may contribute to the confirmation of SOZ. For example, Motoi $\mathrm{H}$ et al. reported that observation of strong phase-amplitude coupling of HFOs with $3-4 \mathrm{~Hz}$ delta waves in non-resected tissues indicates that class I outcomes are less likely to occur [54]. EEG signal below the delta band is often defined as the DC shifts, which would be of great value to SOZ localization [55]. However, few studies have directly addressed the value of cooccurrence of HFOs with DC shifts, and one study by simultaneous evaluation revealed partial spatial overlap between ictal HFOs and DC shifts in SOZ [56].

It is well known that patients with spikes generated in multiple brain regions are less likely to become seizurefree after operation than patients with well located spikes [57]. Therefore, new measurements for epileptogenicity are required in addition to the spikes in intracranial EEG. In the presurgical decisions, the HFO zones might could replace the irritative zone, especially when the irritative zone is widespread.

\section{Localization and lateralization of $\mathrm{SOZ}$ in patients with symptomatic generalized epilepsy}

In symptomatic generalized seizures such as epileptic spasms, myoclonic seizures, tonic seizures, atonic seizures, and atypical absence seizures, the difficulty in localizing SOZ comes from early seizure spread or widespread spikes during seizure onset [58]. However, HFOs can narrow down the range of resection through ripples and fast ripples which occur dozens to hundreds of milliseconds earlier in several electrodes. Most of the epileptogenic zones are located in the posterior frontal central region and posterior temporal-parietal-occipital region [59]. Patients could be improved by focal resection of the epileptogenic zone using HFOs localization.

It was observed that cortical HFOs were related to the epileptic spasms (ESs) corresponding to the scalp fast oscillations $(40-150 \mathrm{~Hz})[60,61]$. Slow waves pattern is the characteristic of ictal EEG in ES [62]. It was reported that the ictal HFOs in the electrocorticography (ECoG) was time-locked to the slow waves when it is less than 1 $\mathrm{Hz}$ [63]. Asymmetries or local components were also found in the ictal slow waves in symptomatic patients [64]. Pre-ictal of clinical spasm, HFOs began and became quasiperiodic. With the evolution of clinical spasm, HFOs persist in the areas of subclinical onset, but its frequency and power were higher than other areas [60]. Complete resection of the sites showing the earliest augmentation of ictal HFOs or slow wave-coupled fast ripples is predicted to a good surgical outcome $[61,65]$.

Myoclonic seizures are epileptic seizures in which the motor manifestation are myoclonus [66]. Myoclonic seizures often have a widespread seizure onset area and cannot be easily localized, which makes it difficult to perform epilepsy surgery, especially in the absence of correlative focal brain lesions. However, using broadband ECoG time-frequency analysis on myoclonic seizures in patients, the power of ripple bands started to rise $2 \mathrm{~s}$ before the clinical seizure onset, peaked $0.5 \mathrm{~s}$ before the clinical seizure onset. For the ripple band in the resected zone, the power during the preictal period increased significantly compared with the power in the interictal period, and myoclonic seizures without clinical lateralizing signs could be improved by focal resection of the epileptogenic zone [67].

In the ictal EEGs of tonic seizures, the gamma rhythms of $43-101.6 \mathrm{~Hz}$ can be detected by time extension and spectrum analysis of the ictal EEG traces [68]. Therefore, more attention should be paid to clinical applications of HFOs in localization and lateraliztion of $\mathrm{SOZ}$ in patients with symptomatic generalized epilepsy.

\section{Localization and lateralization of SOZ in focal epilepsy}

HFOs can be recorded in the human focal epileptic area, mainly in the originate epileptic area. The presence of 
focal HFOs near the time of seizure onset may be close to the epileptogenic foci in mesial temporal lobe and neocortical seizures.

\section{The value of interictal HFOs in epilepsy}

Interictal HFOs are produced spontaneously and intermittently during resting periods. Studies in children and adults reported that surgical failure is related to a high incidence rate of interictal HFOs in the non-resected tissues during non-REM sleep and general anesthesia. The occurrence rate of all these interictal HFOs is statedependent. They often occur in slow-wave sleep [69-71] and during immobility, but rarely in rapid-eyemovement sleep and active wakefulness.

Experimental and clinical studies of HFOs about mesiotemporal lobe (MTL) epilepsy demonstrated that the rate of fast ripples in epileptic hippocampus is significantly higher. In the epileptic MTL with atrophic hippocampus and amygdala, the frequency of fast ripples is higher than that of the contralateral MTL, so it provides a promising diagnostic index for epileptic areas related to hippocampal sclerosis and subsequent synaptic reorganization [72].

However, the rate of HFOs is increased in the main SOZ (microelectrodes and macroelectrodes) in bitemporal epilepsy [19]. For clinically-suspected bitemporal epilepsy, monitoring HFOs can assist to confirm the laterality of the onset zone when conventional EEG or brain MRI fails to localize. Ripples in the ictal period and fast ripples in the interictal period may have more advantages in laterality localization [73].

\section{The value of ictal HFOs in epilepsy}

The specificity of ictal-onset HFOs to SOZ was stronger than that of interictal HFOs, although they always occur in the similar channels [48, 74]. Ripple oscillations occur more frequently in the SOZ than fast ripple oscillations, and ripples show higher amplitude at the transition from the interictal to the ictal state. In a previous study, researchers used subdural grid recording and deep macroelectrodes in neocortical epileptic patients to calculate spectral power during the initial ictal activity and found a peak of 80 to $120 \mathrm{~Hz}$ at the contact site close to the epileptic focus. The dynamic evolution of ripples is related to an epileptic episode. In addition to ripples, fast ripples also occur before ictal onset, with higher oscillation frequency and shorter duration [75].

In intracranial EEG recordings of patients with focal epilepsy, there are usually two specific seizure onset electrographic types, hypersynchronous periodic spiking with ictal discharges $<2 \mathrm{~Hz}$ and low-voltage fast activity consisting of low-amplitude oscillations $>12 \mathrm{~Hz}$ [76-79]. An animal study of ictal HFOs in mesial temporal seizures displayed that the specific HFO type were related to the two different seizure-onset patterns; ripples ( $>80$ $\mathrm{Hz}$ ) were dominant during low-voltage fast activity seizures, whereas fast ripples $(>250 \mathrm{~Hz})$ were dominant during hypersynchronous periodic spiking seizures [10], and a recent study on human SEEG data by Gotman's group have shown similar results [80].

\section{Surgical outcome prediction and prognosis based on HFOs}

The surgical outcome rely on SOZ determined by ictalonset HFOs is better than low frequencies activity [48, 60,81 . Frequency spectrum analysis above $80 \mathrm{~Hz}$ is helpful to improve the accuracy of preoperative evaluation [82]. It is worth including the ripple and fast ripple zones into a set of clinical data, comparing them with the known lesional, seizure-onset, and irritative zones, and making a weighted decision on the epileptogenic region [22]. Removal of HFO producing areas is associated with good surgical outcomes for drug-resistant epilepsy in children and adults [83].

A previous study employing 21 patients with temporal lobe epilepsy (TLE) and 9 with extratemporal lobe epilepsy (ETLE) showed that the better surgical outcome is correlated with the resection of HFO-generating tissue in the whole cohort and in the TLE subgroup, but not in the ETLE group [22]. Patients with good outcomes (ILAE classes 1-3) had significantly higher ripple ratio between resected and non-resected contacts than patients with poor outcomes (ILAE classes 4-6). Among all tailoring approaches, the presence of fast ripples in post-ECoG has the highest predictive value for seizure recurrence [79]. Therefore, the presence of HFOs after surgery predicts poor seizure outcome [84]. The mean volume of resection does not correlate with the surgical outcome [85].

To sum up, HFOs are proposed as a new biological marker of SOZ both for focal epilepsy and for symptomatic generalized epilepsy. However, there are also different views. Julia Jacobs et al. recently completed a multicenter clinical study on HFOs, they found that HFOs may be less localization-specific for SOZ than the earlier studies have indicated [86]. Another study also suggests that removal of HFO-generating regions does not mean better surgical outcome [84]. Many studies suggest that physiological and pathological HFOs largely overlap in regions and frequencies $[87,88]$. The application of HFOs is limited by the inability of current techniques to distinguish epileptic HFOs, therefore it is necessary to further improve the techniques to better separate physiological and epileptic HFOs.

\section{Clinical applications of physiological HFOs Localizing value of physiological HFOs in brain eloquent cortex}

Physiological HFOs include evoked/elicited and spontaneous HFOs. HFOs in the visual, somatosensory, motor, 
and auditory cortices are typically evoked/elicited by tasks or stimuli [89-92]. Physiological HFOs in the normal brain during resting periods are spontaneous or endogenous HFOs [42, 93-99]. It is difficult to discriminate physiological HFOs occurring spontaneously from pathological HFOs [100]. The current recommendation is that the resection range should not be determined only based on the incidence or spectral frequency band of interictal HFOs [101]. The best way to predict post-operative functional deficits is to check the "functional integrity" of the candidate cortical targets [102]. This integrity is usually evaluated by a combination of neuropsychological testing, functional neuroimaging, pharmacological inactivation (Wada test), and electro-cortical stimulation (ECS). However, none of these methods has been proved to be accurate enough to reliably predict the risks of cognitive impairment caused by surgery. Although ECS is generally regarded as the gold standard for determining eloquent brain areas, it does not trigger interpretable performance in many cortical areas, especially in the associative cortex, which supports high levels of cognition [103-105]. The analysis of intracranial HFO responses in cognitive tasks is a new supplement for ECS to address these limitations.

In most cases, HFOs measured by intracranial EEG can be used as a particularly helpful indicator of cortical activation with sufficient time accuracy to contrast and compare the activated time courses of different language regions [106-108]. A variety of language tasks have been studied with this approach, including visual object (picture) naming, auditory word repetition, sentence comprehension, auditory speech perception tasks, and reading. HFOs can be used to map language functional cortical regions [109]. Moreover, combining HFOs and ECS responses can well identify visual, somatosensory and motor functional cortices. So, it is necessary to use HFOs to map eloquent brain areas in epileptic patients before surgery.

\section{The discrimination between physiological and pathological HFOs}

Physiological ripples result from synchronous inhibitory postsynaptic potentials on pyramidal cells [110]. In experimental settings, physiological HFOs recorded using microelectrodes have specific regional and laminar distribution patterns, and their shape and other properties help distinguish them from epileptic HFOs [111-113]. However, in clinical settings where HFOs are recorded with macroelectrodes, distinguishing physiological HFOs from pathological HFOs remains a huge challenge [114], especially in the ripple band $[58,115]$.

For physiological and pathological ripples, various functions have been proposed, such as sensory information processing [116], replay of neuronal activation sequences for memory consolidation [117], and involvement in seizures [118]. Physiological ripples $(\sim 200 \mathrm{~Hz})$ primarily exists in the CA1 region of the hippocampus and entorhinal cortex [119]. In the dentate gyrus, ripples can be pathological and epileptogenic. There is evidence that the higher-frequency HFOs (e.g. fast ripples, VHFOs) are more epileptogenic than the lower-frequency HFOs (e.g. ripples or gamma) [6,32, 33].

Pathological HFOs are most likely caused by neurons with abnormally synchronous bursting, which cannot be distinguished from healthy ripples by simple frequency analysis. Possible approaches to distinguishing normal from pathological HFOs include their relationship to interictal EEG spikes; responses to perturbation of the system such as sleep-wake cycle, stimulation, and drugs; metabolic differences that can be measured by functional MRI or optical imaging, and their association with areas of atrophy or lesion on MRI [26].

Ferrari-Marinho $\mathrm{T}$ et al. studied the relationship between the rate of HFOs and the underlying pathology, and found that the rate of HFOs is higher in focal cortical dysplasia and mesial temporal sclerosis than in atrophy and tuberous sclerosis [120]. The understanding of the relationship is further advanced by HFO studies in specific epileptic syndromes. Nariai $\mathrm{H}$ et al. found that HFOs in ripple band $(80-200 \mathrm{~Hz})$ from the rolandic area play an important role in the emergence of epileptic symptomatology in epileptic spasms [61]. Ictal scalp HFOs can also be used to explore lesions for symptomatic West syndrome [121]. Tang $L$ et al. suggested that the strength of HFOs can reflect the severity of childhood absence seizures [37]. HFO rates are highest in areas where lesion overlaps with SOZ, but in patients with $\mathrm{SOZ}$ outside the lesion, such as in nodular heterotopias, HFO rates are clearly higher in the non-lesional $\mathrm{SOZ}$ than in the inactive lesions.

High-amplitude slow waves during NREM sleep and subtypes of REM sleep facilitate isolation of physiological and pathological HFOs. Epileptogenic HFOs are more likely to be coupled with slow-wave $3-4 \mathrm{~Hz}$ than with slow-wave $0.5-1 \mathrm{~Hz}$, whereas physiological HFOs are more likely associated with slow-wave $0.5-1 \mathrm{~Hz}$ than slow-wave 3-4 Hz during slow-wave sleep [87]. The first sleep cycle seems to be the optimal time to study HFOs in epilepsy because it provides the greatest difference between physiological and pathological ripple rates [122]. Compared with tonic REM sleep, pathological HFOs are suppressed to the greatest extent during phasic REM, while physiological HFOs exhibit the opposite behavior with higher rates during phasic REM compared to tonic REM sleep [123].

\section{Conclusion}

HFOs are a reliable marker of pathological network activity, and should be adopted in the clinical practice to launch more targeted therapeutic methods. However, 
there are still different views among laboratories on the application of HFOs as biomarkers of epileptogenesis and ictogenesis.

\section{Perspective}

Future multicenter studies with large sample sizes and a solid design are needed. Objective methods of HFO analysis can help standardize HFO assessment, which is warranted to demonstrating the utilization of HFOs as a biomarker in epilepsy and to assess disease activity. There are still several aspects to consider for noninvasive use of HFOs to assess disease activity in a large number of patients. First, ensure correct conduction of HFOs detection, as it is critical for optimizing the outcome. Wireless teleconsultation system or other methods should be used to contact HFO experts to maintain the accuracy of HFOs detections in hospitals no matter in developing or developed countries. Second, arrange different healthcare services according to different epilepsies and adjust hospital schedule for different epilepsies. Third, take into account the influencing factors like age and gender, as the pathological significance of HFOs differs in children versus adults and females versus males. Fourth, a rational reimbursement program should be made to compensate patients for making diagnosis by using HFOs. Generally, the assessment of HFOs using noninvasive methods provides a new way to confirm the SOZ and evaluate disease activity in patients with epilepsy.

\begin{abstract}
Abbreviations
Al: Artificial intelligence; ABPE: Atypical benign partial epilepsy; CSWS: Continuous spike-waves during slow-wave sleep; DC: Direct current; ES: Epileptic spasm; ECoG: Electrocorticography; ETLE: Extratemporal lobe epilepsy; ECS: Electro-cortical stimulation; HFOs: High frequency oscillations; LFP: Local field potential; LKS: LandauKleffner syndrome; MTL: Mesial temporal lobe; MRI: Magnetic resonance imaging; MEG: Magnetoencephalography; SSAE: Stacked sparse autoencoder; SOZ: Seizure onset zone; TLE: Temporal lobe epilepsy; VHFOs: Very high frequency oscillations
\end{abstract}

\section{Acknowledgements}

We thank Tao Han, Zhexue Xu, Chengtian Zhao, Minjing Hu for organizing experts to prepare this consensus and for editing the manuscript.

\section{Ethical approval and consent to participate}

Not applicable.

\section{Availability of supporting data}

Not applicable.

\section{Authors' contributions \\ Yuping Wang organized, structured, and initiated the consensus. Dong Zhou, Xiaofeng Yang, Xin Xu, Liankun Ren, Tao Yu, Wenjing Zhou, Xiaoqiu Shao, Zhixian Yang, Shuang Wang, Dezhi Cao, Chunyan Liu, Shang Yeong Kwan, and Jing Xiang completed the writing and revision of this consensus together. All authors read and approved the final manuscript.}

\section{Funding}

This consensus was supported by the National Key R\&D Program (No. 2018YFC1314500) and the National Natural Science Foundation of China (No. 81701297, 81801124).

\section{Consent for publication}

Not applicable.

\section{Competing interests}

The authors declare no conflict of interest.

\section{Author details}

'Department of Neurology, Xuanwu Hospital, Capital Medical University, Beijing 100000, China. ${ }^{2}$ Beijing Key Laboratory of Neuromodulation, Beijing 100000, China. ${ }^{3}$ Center of Epilepsy, Beijing Institute of Brain Disorders, Capital Medical University, Beijing 100000, China. ${ }^{4}$ Department of Neurology, West China Hospital, Sichuan University, Chengdu 610000, China.

${ }^{5}$ Neuroelectrophysiological Laboratory, Xuanwu Hospital, Capital Medical University, Beijing 100000, China. ${ }^{6}$ Department of Neurosurgery, General Hospital of PLA, Beijing 100000, China. ${ }^{7}$ Department of Functional Neurosurgery, Xuanwu Hospital, Capital Medical University, Beijing 100000, China. ${ }^{8}$ Epilepsy Center, Tsinghua University Yuquan Hospital, Beijing 100000, China. ${ }^{9}$ Department of Neurology, Beijing Tiantan Hospital, Capital Medical University, Beijing 100000, China. ${ }^{10}$ China National Clinical Research Center for Neurological Disease NCRC-ND, Beijing 100000, China. " ${ }^{11}$ epartment of Pediatrics, Peking University First Hospital, Beijing 100000, China. ${ }^{12}$ Epilepsy Center, Second Affiliated Hospital, School of Medicine, Zhejiang University, Hangzhou 310000, China. ${ }^{13}$ Department of Neurology, Shenzhen Children's Hospital, Shenzhen 518000, China. ${ }^{14}$ Section of Epilepsy, Neurological Institute, Taipei Veterans General Hospital, Taipei, China. ${ }^{15}$ MEG Center, Cincinnati Children's Hospital Medical Center, Cincinnati, OH 45245, USA. ${ }^{16}$ Division of Neurology, Cincinnati Children's Hospital Medical Center, Cincinnati, $\mathrm{OH} 45245$, USA.

Received: 16 December 2019 Accepted: 12 May 2020

Published online: 12 June 2020

\section{References}

1. Staba RJ, Wilson CL, Bragin A, Fried I, Engel J Jr. Quantitative analysis of high-frequency oscillations $(80-500 \mathrm{~Hz})$ recorded in human epileptic hippocampus and entorhinal cortex. J Neurophysiol. 2002;88(4):1743-52.

2. Frauscher B, Bartolomei F, Kobayashi K, Cimbalnik J, van 't Klooster MA, Rampp S, et al. High-frequency oscillations: the state of clinical research. Epilepsia. 2017;58(8):1316-29.

3. Worrell GA, Jerbi K, Kobayashi K, Lina JM, Zelmann R, Le Van Quyen M. Recording and analysis techniques for high-frequency oscillations. Prog Neurobiol. 2012;98(3):265-78.

4. Jrad N, Kachenoura A, Merlet I, Nica A, Benar CG, Wendling F. Classification of high frequency oscillations in epileptic intracerebral EEG. Conf Proc IEEE Eng Med Biol Soc. 2015;2015:574-7.

5. Usui N, Terada K, Baba K, Matsuda K, Nakamura F, Usui K, et al. Very high frequency oscillations (over $1000 \mathrm{~Hz}$ ) in human epilepsy. Clin Neurophysiol. 2010;121(11):1825-31.

6. Brazdil M, Pail M, Halamek J, Plesinger F, Cimbalnik J, Roman R, et al. Very high-frequency oscillations: novel biomarkers of the epileptogenic zone. Ann Neurol. 2017;82(2):299-310.

7. Dudek FE, Snow RW, Taylor CP. Role of electrical interactions in synchronization of epileptiform bursts. Adv Neurol. 1986;44:593-617.

8. Draguhn A, Traub RD, Schmitz D, Jefferys JG. Electrical coupling underlies high-frequency oscillations in the hippocampus in vitro. Nature. 1998; 394(6689):189-92.

9. Dzhala VI, Staley KJ. Mechanisms of fast ripples in the hippocampus. J Neurosci. 2004;24(40):8896-906.

10. Jiruska P, Alvarado-Rojas C, Schevon CA, Staba R, Stacey W, Wendling F, et al. Update on the mechanisms and roles of high-frequency oscillations in seizures and epileptic disorders. Epilepsia. 2017;58(8):1330-9.

11. Gliske SV, Stacey WC, Lim E, Holman KA, Fink CG. Emergence of narrowband high frequency oscillations from asynchronous, uncoupled neural firing. Int J Neural Syst. 2017;27(1):1650049.

12. Alvarado-Rojas C, Huberfeld G, Baulac M, Clemenceau S, Charpier S, Miles R, et al. Different mechanisms of ripple-like oscillations in the human epileptic subiculum. Ann Neurol. 2015;77(2):281-90.

13. Grenier F, Timofeev I, Steriade M. Focal synchronization of ripples $(80-200 \mathrm{~Hz})$ in neocortex and their neuronal correlates. J Neurophysiol. 2001;86(4):1884-98.

14. Jefferys JG, Menendez de la Prida L, Wendling F, Bragin A, Avoli M, Timofeev I, et al. Mechanisms of physiological and epileptic HFO generation. Prog Neurobiol. 2012;98(3):250-64.

15. Bragin A, Mody I, Wilson CL, Engel J Jr. Local generation of fast ripples in epileptic brain. J Neurosci. 2002;22(5):2012-21. 
16. Bragin A, Wilson CL, Staba RJ, Reddick M, Fried I, Engel J Jr. Interictal highfrequency oscillations $(80-500 \mathrm{~Hz})$ in the human epileptic brain: entorhinal cortex. Ann Neurol. 2002;52(4):407-15.

17. Buzsaki G. Large-scale recording of neuronal ensembles. Nat Neurosci. 2004; 7(5):446-51.

18. Whitmer D, Worrell G, Stead M, Lee IK, Makeig S. Utility of independent component analysis for interpretation of intracranial EEG. Front Hum Neurosci. 2010:4:184

19. Worrell GA, Gardner AB, Stead SM, Hu S, Goerss S, Cascino GJ, et al. Highfrequency oscillations in human temporal lobe: simultaneous microwire and clinical macroelectrode recordings. Brain. 2008;131(4):928-37.

20. Jacobs J, Zijlmans M, Zelmann R, Olivier A, Hall J, Gotman J, et al. Value of electrical stimulation and high frequency oscillations $(80-500 \mathrm{~Hz})$ in identifying epileptogenic areas during intracranial EEG recordings. Epilepsia. 2010;51(4):573-82.

21. Menendez de la Prida L, Staba R, Dian JA. Conundrums of high-frequency oscillations (80-800 Hz) in the epileptic brain. J Clin Neurophysiol. 2015;32(3):207-19.

22. Jacobs J, Zijlmans M, Zelmann R, Chatillon CE, Hall J, Olivier A, et al. Highfrequency electroencephalographic oscillations correlate with outcome of epilepsy surgery. Ann Neurol. 2010;67(2):209-20.

23. Pizzo F, Frauscher B, Ferrari-Marinho T, Amiri M, Dubeau F, Gotman J. Detectability of fast ripples $(>250 \mathrm{~Hz}$ ) on the scalp EEG: a proof-of-principle study with subdermal electrodes. Brain Topogr. 2016;29(3):358-67.

24. Zelmann R, Lina JM, Schulze-Bonhage A, Gotman J, Jacobs J. Scalp EEG is not a Blur: It Can See High Frequency Oscillations Although Their Generators are Small. Brain Topography. 2014;27(5):683-704.

25. Shibata T, Kobayashi K. Epileptic high-frequency oscillations in scalp electroencephalography. Acta Med Okayama. 2018;72(4):325-9.

26. Jacobs J, Staba R, Asano E, Otsubo H, Wu JY, Zijlmans M, et al. High-frequency oscillations (HFOs) in clinical epilepsy. Prog Neurobiol. 2012;98(3):302-15.

27. Benar CG, Chauviere L, Bartolomei F, Wendling F. Pitfalls of high-pass filtering for detecting epileptic oscillations: a technical note on "false" ripples. Clin Neurophysiol. 2010;121(3):301-10

28. Kobayashi K, Watanabe Y, Inoue T, Oka M, Yoshinaga H, Ohtsuka Y. Scalprecorded high-frequency oscillations in childhood sleep-induced electrical status epilepticus. Epilepsia. 2010;51(10):2190-4.

29. Qian P, Li H, Xue J, Yang Z. Scalp-recorded high-frequency oscillations in atypical benign partial epilepsy. Clin Neurophysiol. 2016;127(10):3306-13.

30. Gong P, Xue J, Qian P, Yang H, Liu X, Cai L, et al. Scalp-recorded highfrequency oscillations in childhood epileptic encephalopathy with continuous spike-and-wave during sleep with different etiologies. Brain Dev. 2018:40(4):299-310.

31. Cao D, Chen Y, Liao J, Nariai H, Li L, Zhu Y, et al. Scalp EEG high frequency oscillations as a biomarker of treatment response in epileptic encephalopathy with continuous spike-and-wave during sleep (CSWS) Seizure. 2019;71:151-7.

32. Xiang J, Wang Y, Chen Y, Liu Y, Kotecha R, Huo X, et al. Noninvasive localization of epileptogenic zones with ictal high-frequency neuromagnetic signals. J Neurosurg Pediatr. 2010;5(1):113-22.

33. Xiang J, Liu Y, Wang Y, Kirtman EG, Kotecha $R$, Chen $Y$, et al. Frequency and spatial characteristics of high-frequency neuromagnetic signals in childhood epilepsy. Epileptic Disord. 2009;11(2):113-25.

34. Xiang J, Holowka S, Qiao H, Sun B, Xiao Z, Jiang Y, et al. Automatic localization of epileptic zones using magnetoencephalography. Neurol Clin Neurophysiol. 2004;2004:98

35. Miao A, Xiang J, Tang L, Ge H, Liu H, Wu T, et al. Using ictal high-frequency oscillations $(80-500 \mathrm{~Hz})$ to localize seizure onset zones in childhood absence epilepsy: a MEG study. Neurosci Lett. 2014;566:21-6.

36. Miao A, Tang L, Xiang J, Guan Q, Ge H, Liu H, et al. Dynamic magnetic source imaging of absence seizure initialization and propagation: a magnetoencephalography study. Epilepsy Res. 2014;108(3):468-80.

37. Tang L, Xiang J, Huang S, Miao A, Ge H, Liu H, et al. Neuromagnetic highfrequency oscillations correlate with seizure severity in absence epilepsy. Clin Neurophysiol. 2016;127(2):1120-9

38. Xiang J, Korman A, Samarasinghe KM, Wang X, Zhang F, Qiao H, et al. Volumetric imaging of brain activity with spatial-frequency decoding of neuromagnetic signals. J Neurosci Methods. 2015;239:114-28.

39. Xiang J, Luo Q, Kotecha R, Korman A, Zhang F, Luo H, et al. Accumulated source imaging of brain activity with both low and high-frequency neuromagnetic signals. Front Neuroinform. 2014;8(57):57.

40. Guo J, Yang K, Liu H, Yin C, Xiang J, Li H, et al. A stacked sparse autoencoder-based detector for automatic identification of Neuromagnetic high frequency oscillations in epilepsy. IEEE Trans Med Imaging. 2018;37(11): 2474-82.

41. Hillebrand A, Singh KD, Holliday IE, Furlong PL, Barnes GR. A new approach to neuroimaging with magnetoencephalography. Hum Brain Mapp. 2005: 25(2):199-211.

42. Blanco JA, Stead M, Krieger A, Stacey W, Maus D, Marsh E, et al. Data mining neocortical high-frequency oscillations in epilepsy and controls. Brain. 2011; 134(10):2948-59.

43. Zelmann R, Mari F, Jacobs J, Zijlmans M, Chander R, Gotman J. Automatic detector of high frequency oscillations for human recordings with macroelectrodes. Conf Proc IEEE Eng Med Biol Soc. 2010;2010:2329-33.

44. Gliske SV, Irwin ZT, Chestek C, Hegeman GL, Brinkmann B, Sagher O, et al. Variability in the location of high frequency oscillations during prolonged intracranial EEG recordings. Nat Commun. 2018;9(1):2155

45. Gardner AB, Worrell GA, Marsh E, Dlugos D, Litt B. Human and automated detection of high-frequency oscillations in clinical intracranial EEG recordings. Clin Neurophysiol. 2007:118(5):1134-43.

46. Salami P, Levesque M, Gotman J, Avoli M. A comparison between automated detection methods of high-frequency oscillations $(80-500 \mathrm{~Hz})$ during seizures. J Neurosci Methods. 2012;211(2):265-71.

47. Kobayashi K, Jacobs J, Gotman J. Detection of changes of high-frequency activity by statistical time-frequency analysis in epileptic spikes. Clin Neurophysiol. 2009;120(6):1070-7.

48. Zijlmans M, Jacobs J, Kahn YU, Zelmann R, Dubeau F, Gotman J. Ictal and interictal high frequency oscillations in patients with focal epilepsy. Clin Neurophysiol. 2011;122(4):664-71.

49. Kambara T, Sood S, Alqatan Z, Klingert C, Ratnam D, Hayakawa A, et al. Presurgical language mapping using event-related high-gamma activity: The Detroit procedure. Clin Neurophysiol Official J Int Fed Clin Neurophysiol. 2017;129(1):145.

50. Jacobs J, Zelmann R, Jirsch J, Chander R, Dubeau CE, Gotman J. High frequency oscillations $(80-500 \mathrm{~Hz})$ in the preictal period in patients with focal seizures. Epilepsia. 2009;50(7):1780-92.

51. Jacobs J, LeVan P, Chander R, Hall J, Dubeau F, Gotman J. Interictal highfrequency oscillations ( $80-500 \mathrm{~Hz}$ ) are an indicator of seizure onset areas independent of spikes in the human epileptic brain. Epilepsia. 2008;49(11): 1893-907.

52. Jacobs J, Kobayashi K, Gotman J. High-frequency changes during interictal spikes detected by time-frequency analysis. Clin Neurophysiol. 2011;122(1):32-42.

53. Liu S, Gurses C, Sha Z, Quach MM, Sencer A, Bebek N, et al. Stereotyped high-frequency oscillations discriminate seizure onset zones and critical functional cortex in focal epilepsy. Brain. 2018;141(3):713-30.

54. Motoi H, Miyakoshi M, Abel TJ, Jeong JW, Nakai Y, Sugiura A, et al. Phaseamplitude coupling between interictal high-frequency activity and slow waves in epilepsy surgery. Epilepsia. 2018;59(10):1954-65.

55. Kanazawa K, Matsumoto R, Imamura H, Matsuhashi M, Kikuchi T, Kunieda T, et al. Intracranially recorded ictal direct current shifts may precede high frequency oscillations in human epilepsy. Clin Neurophysiol. 2015;126(1):47-59.

56. Modur PN, Scherg M. Intracranial broadband EEG analysis and surgical outcome: case report. Clin Neurophysiol. 2009;120(6):1220-4.

57. Bautista RE, Cobbs MA, Spencer DD, Spencer SS. Prediction of surgical outcome by interictal epileptiform abnormalities during intracranial EEG monitoring in patients with extrahippocampal seizures. Epilepsia. 1999:40(7):880-90.

58. Zijlmans M, Jiruska P, Zelmann R, Leijten FS, Jefferys JG, Gotman J. Highfrequency oscillations as a new biomarker in epilepsy. Ann Neurol. 2012 71(2):169-78.

59. Yu T, Zhang G, Wang $Y$, Ni D, Qiao L, Du W, et al. Surgical treatment for patients with symptomatic generalised seizures due to brain lesions. Epilepsy Res. 2015;112:92-9.

60. Akiyama T, Otsubo H, Ochi A, Ishiguro T, Kadokura G, Ramachandrannair R, et al. Focal cortical high-frequency oscillations trigger epileptic spasms: confirmation by digital video subdural EEG. Clin Neurophysiol. 2005;116(12):2819-25.

61. Nariai H, Nagasawa T, Juhasz C, Sood S, Chugani HT, Asano E. Statistical mapping of ictal high-frequency oscillations in epileptic spasms. Epilepsia. 2011;52(1):63-74.

62. Fusco L, Vigevano F. Ictal clinical electroencephalographic findings of spasms in west syndrome. Epilepsia. 1993;34(4):671-8.

63. Akiyama T, Chan DW, Go CY, Ochi A, Elliott IM, Donner EJ, et al. Topographic movie of intracranial ictal high-frequency oscillations with seizure semiology: epileptic network in Jacksonian seizures. Epilepsia. 2011; 52(1):75-83. 
64. Kobayashi K, Oka M, Inoue T, Ogino T, Yoshinaga H, Ohtsuka Y. Characteristics of slow waves on EEG associated with epileptic spasms. Epilepsia. 2005;46(7):1098-105.

65. limura Y, Jones K, Takada L, Shimizu I, Koyama M, Hattori K, et al. Strong coupling between slow oscillations and wide fast ripples in children with epileptic spasms: investigation of modulation index and occurrence rate. Epilepsia. 2018;59(3):544-54.

66. Caviness JN. Myoclonus. Mayo Clin Proc. 1996;71(7):679-88.

67. Sun Y, Zhang G, Zhang X, Yan X, Li L, Xu C, et al. Time-frequency analysis of intracranial EEG in patients with myoclonic seizures. Brain Res. 2016;1652: 119-26.

68. Kobayashi K, Inoue T, Watanabe Y, Oka M, Endoh F, Yoshinaga H, et al. Spectral analysis of EEG gamma rhythms associated with tonic seizures in Lennox-Gastaut syndrome. Epilepsy Res. 2009;86(1):15-22.

69. Bagshaw AP, Jacobs J, LeVan P, Fo D, Gotman J. Effect of sleep stage on interictal high-frequency oscillations recorded from depth macroelectrodes in patients with focal epilepsy. Epilepsia. 2009:50(4):617-28.

70. Staba RJ, Wilson CL, Bragin A, Jhung D, Fried I, Engel J. High-frequency oscillations recorded in human medial temporal lobe during sleep. Ann Neurol. 2004;56(1):108-15.

71. Jirsch DJ. High-frequency oscillations during human focal seizures. Brain. 2006;129(6):1593-608.

72. Crepon B, Navarro V, Hasboun D, Clemenceau S, Martinerie J, Baulac M, et al. Mapping interictal oscillations greater than $200 \mathrm{~Hz}$ recorded with intracranial macroelectrodes in human epilepsy. Brain. 2010;133(1):33-45.

73. Liu C, Zhang R, Zhang G, Yu T, Tai J, Du W, et al. High frequency oscillations for lateralizing suspected bitemporal epilepsy. Epilepsy Res. 2016;127:233-40.

74. Zijlmans M, Jacobs J, Zelmann R, Dubeau F, Gotman J. High-frequency oscillations mirror disease activity in patients with epilepsy. Neurology. 2009; 72(11):979-86.

75. Kobayashi K, Agari T, Oka M, Yoshinaga H, Date I, Ohtsuka Y, et al. Detection of seizure-associated high-frequency oscillations above $500 \mathrm{~Hz}$. Epilepsy Res. 2010;88(2-3):139-44

76. Velasco AL, Wilson $\mathrm{CL}$, Babb TL, Engel J Jr. Functional and anatomic correlates of two frequently observed temporal lobe seizure-onset patterns. Neural Plast. 2000;7(1-2):49-63.

77. Ogren JA, Bragin A, Wilson CL, Hoftman GD, Lin JJ, Dutton RA, et al. Threedimensional hippocampal atrophy maps distinguish two common temporal lobe seizure-onset patterns. Epilepsia. 2009;50(6):1361-70.

78. Perucca P, Dubeau F, Gotman J. Intracranial electroencephalographic seizure-onset patterns: effect of underlying pathology. Brain. 2014;137(1):183-96.

79. Memarian N, Madsen SK, Macey PM, Fried I, Engel J Jr, Thompson PM, et al. Ictal depth EEG and MRI structural evidence for two different epileptogenic networks in mesial temporal lobe epilepsy. PLoS One. 2015;10(4):e0123588.

80. Ferrari-Marinho T, Perucca P, Dubeau F, Gotman J. Intracranial EEG seizure onset-patterns correlate with high-frequency oscillations in patients with drug-resistant epilepsy. Epilepsy Res. 2016;127:200-6.

81. Grinenko O, Li J, Mosher JC, Wang IZ, Bulacio JC, Gonzalez-Martinez J, et al. A fingerprint of the epileptogenic zone in human epilepsies. Brain. 2018; 141(1):117-31.

82. Jiruska P, Tomasek M, Netuka D, Otahal J, Jefferys JG, Li X, et al. Clinical impact of a high-frequency seizure onset zone in a case of bitemporal epilepsy. Epileptic Disord. 2008;10(3):231-8.

83. Wu JY, Sankar R, Lerner JT, Matsumoto JH, Vinters HV, Mathern GW. Removing interictal fast ripples on electrocorticography linked with seizure freedom in children. Neurology. 2010;75(19):1686-94.

84. van 't Klooster MA, van Klink NEC, Zweiphenning W, FSS L, Zelmann R, Ferrier $\mathrm{CH}$, et al. Tailoring epilepsy surgery with fast ripples in the intraoperative electrocorticogram. Ann Neurol. 2017:81(5):664-76.

85. Haegelen C, Perucca P, Chatillon CE, Andrade-Valenca L, Zelmann R, Jacobs $J$, et al. High-frequency oscillations, extent of surgical resection, and surgical outcome in drug-resistant focal epilepsy. Epilepsia. 2013;54(5):848-57.

86. Jacobs J, Wu JY, Perucca P, Zelmann R, Mader M, Dubeau F, et al. Removing high-frequency oscillations: a prospective multicenter study on seizure outcome. Neurology. 2018;91(11):e1040-e52

87. Nonoda Y, Miyakoshi M, Ojeda A, Makeig S, Juhasz C, Sood S, et al. Intericta high-frequency oscillations generated by seizure onset and eloquent areas may be differentially coupled with different slow waves. Clin Neurophysiol. 2016;127(6):2489-99.

88. Engel J Jr, Bragin A, Staba R, Mody I. High-frequency oscillations: what is normal and what is not? Epilepsia. 2009;50(4):598-604.
89. Huo X, Xiang J, Wang Y, Kirtman EG, Kotecha R, Fujiwara H, et al. Gamma oscillations in the primary motor cortex studied with MEG. Brain and Development. 2010;32(8):619-24.

90. Chen Y, Xiang J, Kirtman EG, Wang Y, Kotecha R, Liu Y. Neuromagnetic biomarkers of visuocortical development in healthy children. Clin Neurophysiol. 2010;121(9):1555-62.

91. Xiang J, Holowka S, Sharma R, Hunjan A, Otsubo H, Chuang S. Volumetric localization of somatosensory cortex in children using synthetic aperture magnetometry. Pediatr Radiol. 2003;33(5):321-7.

92. Xiang J, Wilson D, Otsubo H, Ishii R, Chuang S. Neuromagnetic spectral distribution of implicit processing of words. Neuroreport. 2001;12(18):3923-7.

93. Fukushima M, Saunders RC, Leopold DA, Mishkin M, Averbeck BB. Spontaneous high-gamma band activity reflects functional organization of auditory cortex in the awake macaque. Neuron. 2012;74(5):899-910.

94. Melani F, Zelmann R, Mari F, Gotman J. Continuous high frequency activity: a peculiar SEEG pattern related to specific brain regions. Clin Neurophysiol. 2013:124(8):1507-16.

95. Nagasawa T, Juhasz C, Rothermel R, Hoechstetter K, Sood S, Asano E. Spontaneous and visually driven high-frequency oscillations in the occipital cortex: intracranial recording in epileptic patients. Hum Brain Mapp. 2012;33(3):569-83.

96. Wang S, Wang IZ, Bulacio JC, Mosher JC, Gonzalez-Martinez J, Alexopoulos $A V$, et al. Ripple classification helps to localize the seizure-onset zone in neocortical epilepsy. Epilepsia. 2013;54(2):370-6.

97. Alkawadri R, Gaspard N, Goncharova II, Spencer DD, Gerrard JL, Zaveri H, et al. The spatial and signal characteristics of physiologic high frequency oscillations. Epilepsia. 2014;55(12):1986-95.

98. van 't Klooster MA, van Klink NE, Leijten FS, Zelmann R, Gebbink TA, Gosselaar $\mathrm{PH}$, et al. Residual fast ripples in the intraoperative corticogram predict epilepsy surgery outcome. Neurology. 2015;85(2):120-8.

99. Schnitzler S, Hartmann CJ, Groiss SJ, Wojtecki L, Schnitzler A, Vesper J, et al. Occurrence of thalamic high frequency oscillations in patients with different tremor syndromes. Clin Neurophysiol. 2018;129(5):959-66.

100. Xiang J, Liu Y, Wang Y, Kotecha R, Kirtman EG, Chen Y, et al. Neuromagnetic correlates of developmental changes in endogenous high-frequency brain oscillations in children: a wavelet-based beamformer study. Brain Res. 2009; 1274:28-39.

101. Gliske SV, Irwin ZT, Davis KA, Sahaya K, Chestek C, Stacey WC. Universal automated high frequency oscillation detector for real-time, long term EEG. Clin Neurophysiol. 2016;127(2):1057-66.

102. Chelune GJ. Hippocampal adequacy versus functional reserve: predicting memory functions following temporal lobectomy. Arch Clin Neuropsychol. 1995;10(5):413-32

103. Halgren E, Babb TL, Crandall PH. Activity of human hippocampal formation and amygdala neurons during memory testing. Electroencephalogr Clin Neurophysiol. 1978;45(5):585-601.

104. Halgren E, Walter RD, Cherlow DG, Crandall PH. Mental phenomena evoked by electrical stimulation of the human hippocampal formation and amygdala. Brain. 1978:101(1):83-117.

105. David O, Bastin J, Chabardes S, Minotti L, Kahane P. Studying network mechanisms using intracranial stimulation in epileptic patients. Front Syst Neurosci. 2010;4:148

106. Canolty RT, Soltani M, Dalal SS, Edwards E, Dronkers NF, Nagarajan SS, et al. Spatiotemporal dynamics of word processing in the human brain. Front Neurosci. 2007:1(1):185-96.

107. Crone NE, Boatman D, Gordon B, Hao L. Induced electrocorticographic gamma activity during auditory perception. Brazier award-winning article, 2001. Clin Neurophysiol. 2001;112(4):565-82.

108. Edwards E, Soltani M, Deouell LY, Berger MS, Knight RT. High gamma activity in response to deviant auditory stimuli recorded directly from human cortex. J Neurophysiol. 2005;94(6):4269-80.

109. Lachaux JP, Axmacher N, Mormann F, Halgren E, Crone NE. High-frequency neural activity and human cognition: past, present and possible future of intracranial EEG research. Prog Neurobiol. 2012;98(3):279-301.

110. Ylinen A, Bragin A, Nadasdy Z, Jando G, Szabo I, Sik A, et al. Sharp waveassociated high-frequency oscillation $(200 \mathrm{~Hz})$ in the intact hippocampus: network and intracellular mechanisms. J Neurosci. 1995;15(1):30-46.

111. Bragin A, Wilson CL, Engel J Jr. Voltage depth profiles of high-frequency oscillations after kainic acid-induced status epilepticus. Epilepsia. 2007:48(Suppl 5):35-40.

112. Bragin A, Engel J Jr, Wilson CL, Fried I, Buzsaki G. High-frequency oscillations in human brain. Hippocampus. 1999;9(2):137-42.

113. Bragin A, Engel J Jr, Wilson CL, Fried I, Mathern GW. Hippocampal and entorhinal cortex high-frequency oscillations (100--500 Hz) in human 
epileptic brain and in kainic acid--treated rats with chronic seizures. Epilepsia. 1999;40(2):127-37.

114. Cimbalnik J, Brinkmann B, Kremen V, Jurak P, Berry B, Gompel JV, et al. Physiological and pathological high frequency oscillations in focal epilepsy. Ann Clin Transl Neurol. 2018;5(9):1062-76.

115. Frauscher B, von Ellenrieder N, Zelmann R, Rogers C, Nguyen DK, Kahane P, et al. High-frequency oscillations in the Normal human brain. Ann Neurol. 2018;84(3):374-85.

116. Jones MS, Barth DS. Spatiotemporal organization of fast $(>200 \mathrm{~Hz})$ electrical oscillations in rat vibrissa/barrel cortex. J Neurophysiol. 1999;82(3):1599-609.

117. Nadasdy Z, Hirase H, Czurko A, Csicsvari J, Buzsaki G. Replay and time compression of recurring spike sequences in the hippocampus. J Neurosci. 1999;19(21):9497-507.

118. Traub RD, Whittington MA, Buhl EH, LeBeau FE, Bibbig A, Boyd S, et al. A possible role for gap junctions in generation of very fast EEG oscillations preceding the onset of, and perhaps initiating, seizures. Epilepsia. 2001;42(2): $153-70$.

119. Csicsvari J, Hirase H, Czurko A, Mamiya A, Buzsaki G. Fast network oscillations in the hippocampal CA1 region of the behaving rat. J Neurosci. 1999;19(16):RC20.

120. Ferrari-Marinho T, Perucca P, Mok K, Olivier A, Hall J, Dubeau F, et al. Pathologic substrates of focal epilepsy influence the generation of highfrequency oscillations. Epilepsia. 2015;56(4):592-8.

121. Iwatani Y, Kagitani-Shimono K, Tominaga K, Okinaga T, Kishima H, Kato A, et al. Ictal high-frequency oscillations on scalp EEG recordings in symptomatic west syndrome. Epilepsy Res. 2012;102(1-2):60-70.

122. von Ellenrieder N, Dubeau F, Gotman J, Frauscher B. Physiological and pathological high-frequency oscillations have distinct sleep-homeostatic properties. Neurolmage Clin. 2017;14:566-73.

123. Frauscher B, von Ellenrieder N, Dubeau F, Gotman J. EEG desynchronization during phasic REM sleep suppresses interictal epileptic activity in humans. Epilepsia. 2016;57(6):879-88.

Ready to submit your research? Choose BMC and benefit from:

- fast, convenient online submission

- thorough peer review by experienced researchers in your field

- rapid publication on acceptance

- support for research data, including large and complex data types

- gold Open Access which fosters wider collaboration and increased citations

- maximum visibility for your research: over $100 \mathrm{M}$ website views per year

At $\mathrm{BMC}$, research is always in progress.

Learn more biomedcentral.com/submissions 